\title{
Tenth International PCB Workshop: 50 years of PCB research, new approaches and discoveries and still so much more to learn
}

\author{
Larry W. Robertson ${ }^{1}$
}

Published online: 22 February 2020

(C) Springer-Verlag GmbH Germany, part of Springer Nature 2020

The Tenth International PCB Workshop took place at the Hotel Q Plus Conference Centre, Krakow, Poland, during August 27-31, 2018. It was held in conjunction with the Dioxin 2018 International Symposium on Halogenated Persistent Organic Pollutants. Altogether, the meetings attracted 728 participants.

The program included four plenary presentations, four workshop sessions, and one poster session. Thirty nine experts on PCBs presented at the workshop sessions on the Stockholm Convention, Sources, Exposures, Inventories and Actions to Reduce Exposures; Evolving Approaches to Assessing Exposures and Health Risks from Environmental Chemical Mixtures; Novel Studies on PCB Toxicity and Mechanisms Action; and PCB regulations for Health Protection: Recent Actions, Ongoing Initiatives, and Future Perspectives.

In total, presenters represented fourteen different countries from around the world. The local organizing committee consisted of nineteen members from twelve different countries. The PCB Workshop was chaired by Dr. Larry Robertson, director of the Iowa Superfund Research Program for 12 years, and Dr. Magdalena Urbaniak, associate professor at European Regional Centre for Ecohydrology of the Polish Academy of Sciences. Dr. Robertson was also co-chair of the Dioxin 2018 Symposium where he presented a plenary talk.

Session 1 led by Dr. Niklas Johansson (Sweden) and Dr. Keri Hornbuckle (University of Iowa) focused on international activities in the frame of the Stockholm

Responsible editor: Philippe Garrigues

Larry W. Robertson

larry-robertson@uiowa.edu

1 Department of Occupational \& Environmental Health, College of Public Health, The University of Iowa, Iowa City, Iowa, USA
Convention to eliminate PCBs by 2028 to limit human and environmental impact of PCBs and provided insights into challenges and progress of global management of PCB stocks and waste.

Session 2 on Evolving Approaches to Assessing Exposures and Health Risks from Environmental Chemical Mixtures was chaired by Dr. Geniece Lehmann (US EPA) and Dr. Mattias Oberg (Karolinska Institute). Special challenges and approaches to risk assessment of environmental mixtures were presented.

Session 3 on Novel Studies on PCB Toxicity and Mechanisms Action was led by Dr. Mirek Machala and Dr. Michael Duffel. Dr. Michala is head of the Department of Chemistry and Toxicology at the Veterinary Research Institute in the Czech Republic. Dr. Duffel is professor and associate dean for Research and Graduate Programs, University of Iowa College of Pharmacy.

Session 4 on PCB regulations for Health Protection: Recent Actions, Ongoing Initiatives, and Future Perspectives was led by Dr. Helen Hakansson (Sweden) and Dr. Vince Cogliano (USEPA). Dr. Hakansson is professor emeritus in the Department of Environmental Medicine at the Karolinska Institute. Dr. Cogliano is a health risk assessment scientist at the USEPA.

On the following pages are nine publications arising out of the workshop. They highlight advances in the synthesis and detection of PCBs and their metabolites, occurrence in environmental various matrices, and their biological effects. Of the variety of new information presented at the meeting and in the following pages, here are three highlights that may be of interest:

Research from the laboratory of Professor Michael Duffel of the University of Iowa has provided valuable new information that will enable assessment of the potential for sulfated metabolites to be significant contributors to the toxicities observed upon exposure to airborne PCBs. His workshop presentation on the roles 
of sulfation in the transport and toxicity of airborne PCBs highlighted the isoforms of cytosolic sulfotransferases that catalyze the congener-specific formation of PCB sulfates from corresponding hydroxylated $\mathrm{PCBs}$ (OH-PCBs) and a microsomal sulfatase catalyzing the selective hydrolysis of PCB sulfates to $\mathrm{OH}-$ PCBs. The same enzymes play key roles in the transport and function of a major physiological steroid hormone, dehydroepiandrosterone (DHEA). Following the sulfation of DHEA, DHEA sulfate is released into the serum, transported through binding to serum proteins, taken up by other tissues, and then converted to DHEA through action of the microsomal steroid sulfatase. Similarly, following sulfation of OH-PCBs, $\mathrm{PCB}$ sulfates bind reversibly, with congener-specific affinity, to serum proteins such as human serum albumin and the thyroid hormone transport protein transthyretin. Congener- and cell-selective uptake of PCB sulfates as well as intracellular sulfatase-catalyzed hydrolysis to the corresponding OH-PCBs have also been observed. Thus, it was proposed that a dynamic interchange between $\mathrm{OH}-\mathrm{PCBs}$ and $\mathrm{PCB}$ sulfates occurs that is analogous to the synthesis, transport, cellular uptake, and intracellular hydrolysis of DHEA sulfate. This process may provide a previously unrecognized mechanism for prolonged exposure to $\mathrm{OH}-\mathrm{PCBs}$ that may have important toxicological consequences.

Professor Al Klingelhutz of the University of Iowa is studying the effects of PCBs on adipose tissue. Subcutaneous white adipose tissue is capable of becoming thermogenic in a process that is referred to as "beiging." Beiging is associated with activation of the uncoupling protein, $\mathrm{UCP} 1$, and is known to be important for preventing adipose hypertrophy and development of insulin resistance. PCBs accumulate in fat, and it is hypothesized that disruption of adipogenesis and adipocyte function by PCBs may be causative in the development of obesity and diabetes. Professor Klingelhutz's laboratory developed immortal human subcutaneous preadipocytes that, when differentiated, are capable of beiging. Preadipocytes that were treated with PCB126, followed by differentiation, were suppressed in their ability to activate UCP1 upon $\beta$-adrenergic stimulation with norepinephrine (NE), demonstrating a block in the beiging response. Treatment of preadipocytes with another known endogenous AhR agonist, indoxyl sulfate (IS), followed by differentiation also blocked the NEstimulated upregulation of UCP1. Knockdown of the aryl hydrocarbon receptor (AhR) caused the preadipocytes to be refractory to PCB126 and IS effects. The chemical AhR antagonist, CH223191, was effective at preventing the effects of PCB126 but not IS, indicating AhR ligand specificity of $\mathrm{CH} 223191$. Repression of
NE-induced UCP1 upregulation was also observed when already-differentiated mature adipocytes were treated with PCB126 but not with IS. These results indicate that exposure of preadipocytes to endogenous (IS) or exogenous (PCB126) AhR agonists is effective at blocking them from becoming functional adipocytes that are capable of the beiging response. Mature adipocytes may have differential responses. This finding suggests a mechanism by which dioxin-like PCBs such as PCB126 could lead to disruption in energy homeostasis, potentially leading to obesity and diabetes.

Professor Pamela Lein of the University of California Davis conducted a review of the literature on the neurologic effects of PCBs and presented data from her mechanistic studies of PCB developmental neurotoxicity. She concludes that PCBs remain a significant risk to human health. A primary target of concern is the developing brain. Epidemiological studies link PCB exposures in utero or during infancy to increased risk of neuropsychiatric deficits in children. Nonclinical studies of legacy congeners found in PCB mixtures synthesized prior to the ban on $\mathrm{PCB}$ production suggest that nondioxin-like (NDL) congeners are predominantly responsible for the developmental neurotoxicity associated with PCB exposures. Mechanistic studies suggest that NDL PCBs alter neurodevelopment via ryanodine receptor-dependent effects on dendritic arborization. Lightly chlorinated congeners, which were not present in the industrial mixtures synthesized prior to the ban on PCB production, have emerged as contemporary environmental contaminants, but there is a paucity of data regarding their potential developmental neurotoxicity. PCB 11, a prevalent contemporary congener, is found in the serum of children and their mothers, as well as in the serum of pregnant women at increased risk for having a child diagnosed with a neurodevelopmental disorder (NDD). Recent data from the Lein laboratory demonstrates that PCB 11 modulates neuronal morphogenesis via mechanisms that are convergent with and divergent from those implicated in the developmental neurotoxicity of legacy NDL PCBs. Professor Lein's review summarizes these data and discusses their relevance to adverse neurodevelopmental outcomes in humans.

PCBs have a broad range of targets of toxicity, and even though well-studied, research on PCBs continues to provide surprises.

Funding information Funding for the Tenth International PCB Workshop was made possible (in part) by R13 ES029824 from the National Institute of Environmental Health Sciences and by support from the Iowa Superfund Research Program (P42 ES013661). 


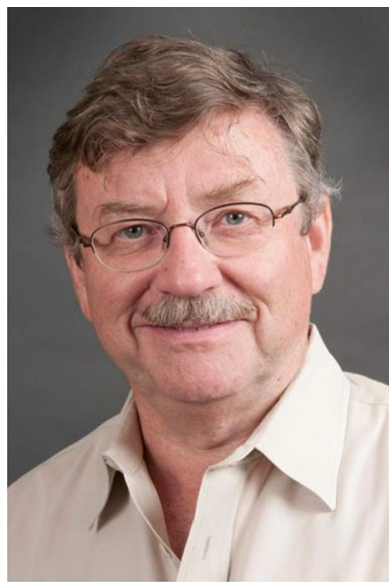

Larry W. Robertson, MPH, $\mathrm{PhD}$, ATS is a professor of toxicology in the Department of Occupational \& Environmental Health, College of Public Health at the University of Iowa. Dr. Robertson is the director of the Interdisciplinary Graduate Program in Human Toxicology, a toxicology training program with 27 full-time doctoral students and over 30 faculty members. Dr. Robertson has published more than 290 original research papers describing the toxicity of polyhalogenated hydrocarbons. 